have since fallen to the discovery that short RNA chains can in vitro and may in vivo act as primers for the self replication of double and single stranded DNAs by DNA polymerases which elongate DNA chains in the $5^{\prime}-3^{\prime}$ direction, polymerizing deoxy nucleotide triphosphates onto $3^{\prime}$ hydroxy groups of primers.

The existence of RNA dependent DNA polymerases (reverse transcriptases) which can use RNA templates to make DNA was, of course, predicted by Temin to account for the curious biology of the RNA tumour viruses, but their potential usefulness to cells as well as these viruses is obvious enough; such enzymes, for example, provide, in theory at least, a way of amplifying any particular gene of which the RNA product of transcription is available. The classic example of gene amplification is the amplification of the ribosomal RNA genes during oogenesis in Amphibia and some insects, and as Crippa and Tochini-Valentini were quick to show in Xenopus oocytes this particular amplification is inhibited by a derivative of rifampicin, AF/ABDMP, which also inhibits the reverse transcriptase of RNA tumour viruses.

Both Crippa and Tochini-Valentini are clearly still enamoured of the idea that amplification of the ribosomal RNA genes depends on reverse transcription and with good reason, for Brown and Tochini-Valentini (Proc. US Nat. Acad. Sci., 69, 1746; 1972) have isolated from the ovaries of tadpoles in metamorphosis newly synthesized ribosomal DNA complexed with a transscript of the ribosomal RNA genes such that the sedimentation of the DNA is sensitive to RNAase. They have also shown that the RNA moiety of this complex can be used as a template for DNA synthesis by a DNA polymerase endogenous to the Xenopus cells in a reaction which is sensitive to $\mathrm{AF} /$ ABDMP. At the same time Mahdavi and Crippa (ibid., 1749) have also isolated this ribosomal DNA ribosomal RNA complex and point out that the RNA fraction contains not only the mature $18 \mathrm{~S}$ and $28 \mathrm{~S}$ ribosomal RNA sequences but also the so-called spacer sequence which links these two genes but is not transcribed by RNA polymerase. All these observations constitute compelling if circumstantial evidence for the claim that ribosomal RNA genes in Xenopus are amplified by reverse transcription.

But irrespective of whether RNA chains have a template function during gene amplification, evidence that short RNA chains act as primers for DNA self replication in microorganisms is fast accumulating in several quarters. For one thing DNA replication in Escherichia coli seems to be sensitive to inhibitors of transcription, and as
Keller has recently shown in vitro at least RNA can prime the synthesis of $\phi X 174$ DNA. Now Sugino, Hirose and Okazaki (ibid., 1863) have entered the lists by showing that the small strands of DNA-the famous Okazaki pieces which are made when $E$. coli cells are fed very short pulses of ${ }^{3} \mathrm{H}$-thymidine - have a buoyant density in caesium sulphate greater than that expected of single stranded DNA. This and other observations lead Sugino et al. to the conclusion that Okazaki pieces, precursors of the nascent daughter DNA strands, are synthesized on RNA primer chains so that at early times they comprise covalently linked RNADNA ; the RNA primer is presumably digested before the short DNA strand is ligated to the nascent chain.

Baltimore and his colleagues reported, of course, last year that reverse transcriptase from tumour viruses initiates the synthesis of DNA on RNA templates at the $3^{\prime}$ hydroxy terminus of an RNA primer. The odds on very short RNA chains being universal primers of all DNA synthesis (except repair synthesis), irrespective of the nature of the template strand, are clearly shortening.

\section{POPULATION ECOLOGY Reproductive Sirategies}

from a Correspondent

Charles Darwin observed that some insects lay many eggs and others but a few, yet this does not seem to be related to their abundance as adults. Ecologists are still attempting to develop a general theory broad enough to explain why some organisms devote a higher proportion of their energy and material to reproduction than do others. This balance between allocation of resources to reproductive functions and to maintenance of the adult organism is one component of the organism's reproductive strategy. It is discussed in detail by Gadgil and Solbrig (Amer. Nat., 106, 14 ; 1972) who account for genetically based differences in this balance between three populations of dandelions (Taraxacum officinale).

MacArthur and Wilson (The Theory of Island Biogeography, Princeton University Press; 1967) have argued that, when a population is increasing in numbers and mortality is low, those genotypes which reproduce fastest will come to predominate. As the population approaches the carrying capacity $(K)$ of its environment, set by resource limitation or by predation, mortality increases, along with selection pressures for characteristics favouring survival (predator avoidance strategies) or increased efficiency in exploiting limited resources (competitive ability). The response to these selection pressures in populations which remain for long periods close to the carrying capacity ( $K$-selected populations) will be an increase in the proportion of resources devoted to increasing survival and efficiency, with a concomitant decrease in resources directly allocated to reproduction. In contrast, where repeated population extinction and recolonization occur, as in "fugitive" species characteristic of temporary habitats, selection for high rate of increase $(r)$ in newly occupied habitats will favour greater allocation of resources to reproduction.

Gadgil and Solbrig use the generaliza-

\title{
Variability of DNA Content of Bacillus Species
}

IN Nature New Biology next Wednesday (August 9) Johnston and Young report an investigation by electronautoradiography of the amount of DNA in individual cells and spores of Bacillus subtilis and $B$. cereus and they conclude that in both cells and spores the amount of DNA is very variable. Estimates of the size of the genomes and DNA contents of Bacillus species are notoriously disparate and to obtain fairly accurate estimates of the amount of DNA in individual spores and cells Johnston and Young had to resort to a very laborious procedure.

They grew cells for ten to twelve generations in a medium containing ${ }^{3} \mathrm{H}$ thymidine and then produced serial sections of labelled cells and spores and made micrographs which were projected and the silver grains were traced and then cut out. As calibration experiments with Escherichia coli revealed, the weight of the grain tracings gives a fairly accurate estimate of the total DNA pre-cell or pre-spore.

When the amount of DNA in Bacillus cells and spores was estimated in this fashion three unexpected findings emerged; first the amount of DNA in different cells of the same species can vary by as much as four to five-fold; second, in individual cells the DNA in a spore is not always a constant fraction of the amount in the sporulating cell; third, the amount of DNA in spores is quite variable.

Johnston and Young estimate the genome of $B$. cereus to be $3 \times 10^{9}$ daltons in which case individual cells may contain from 5-20 genomes whereas spores may have 1-8 genomes, and $B$. subtilis cells may have from 2-9 genomes and $B$. subtilis spores 1-4 genomes. The biochemical basis and evolutionary significance of these striking variations are at present matters for speculation. 